\title{
Absorbance or organization into ankylosis: a microarray analysis of haemarthrosis in a sheep model of temporomandibular joint trauma
}

\author{
Mai-Ning Jiao ${ }^{1,2,3 \dagger}$, Tong-Mei Zhang ${ }^{1,2,3 \dagger}{ }^{\text {, Kun Yang }}{ }^{4}$, Zhao-Yuan Xu ${ }^{2,3}$, Guan-Meng Zhang ${ }^{2,3}$, Yuan-Yuan Tian²,3,
} Hao Liu ${ }^{2,3^{*}}$ and Ying-Bin Yan ${ }^{2,3^{*}}$

\begin{abstract}
Background: Traumatic haemarthrosis was hypothesized to be the etiology of temporomandibular (TMJ) ankylosis. Here, taking haematoma absorbance as a control, we aimed to reveal the molecular mechanisms involved in haematoma organizing into ankylosis using transcriptome microarray profiles.

Material/methods: Disk removal was performed to building haematoma absorbance (HA) in one side of TMJ, while removal of disk and articular fibrous layers was performed to induced TMJ ankylosis through haematoma organization $(\mathrm{HO})$ in the contralateral side in a sheep model. Haematoma tissues harvested at days 1, 4 and 7 postoperatively were examined by histology, and analyzed by Affymetrix OviGene-1_0-ST microarrays. The DAVID were recruited to perform the Gene Ontology and Kyoto Encyclopedia of Genes and Genomes pathway analysis for the different expression genes (DEGs). The DEGs were also typed into protein-protein interaction (PPI) networks to get the interaction data. Six significant genes screened from PPI analysis, were confirmed by real-time PCR.

Results: We found 268, 223 and 17 DEGs at least twofold at days 1, 4 and 7, respectively. At day 1, genes promoting collagen ossification (POSTN, BGN, LUM, SPARC), cell proliferation (TGF- $\beta$ ), and osteogenic differentiation of mesenchymal stem cells (BMP-2) were up-regulated in the $\mathrm{HO}$ side. At day 4, several genes involved in angiogenesis (KDR, FIT1, TEK) shower higher expression in the $\mathrm{HO}$ side. While HA was characterized by a continuous immune and inflammatory reaction.

Conclusions: Our results provide a comprehensive understanding of the role of haematoma in the onset and progress of TMJ ankylosis. The study will contribute to explaining why few injured TMJs ankylose and most do not from the molecular level.
\end{abstract}

Keywords: Temporomandibular ankylosis, Trauma, Hematoma, Microarray analysis, Sheep

*Correspondence: kqlh2013@163.com; yingbinyan@qq.com

${ }^{\dagger}$ Mai-Ning Jiao and Tong-Mei Zhang have contributed equally to this

paper.

${ }^{2}$ Department of Oromaxillofacial-Head and Neck Surgery, Tianjin

Stomatological Hospital, 75 Dagu Road, Heping District, Tianjin 300041,

People's Republic of China

Full list of author information is available at the end of the article

\section{Introduction}

Temporomandibular joint (TMJ) ankylosis refers to bone or fibrous adhesion of the anatomic joint components with the ensuing progressive limitation of mouth opening [1]. Trauma is the most important cause of the disease, account for 31-98\% cases [2]. Although for most patients with ankylosis, the primary trauma was severe enough to 
produce a condylar fracture, traumatic haemarthrosis, rather than the condylar fracture itself, was hypothesized to be the etiology of TMJ ankylosis [3]. The reasons were as follows: 1 . cases with traumatic TMJ ankylosis but without condylar fracture have been reported [3]; 2. some patients developed into TMJ ankylosis after arthroscopy [4]; and 3. only a very few fractured TMJs ankylosed $(0.4-2 \%)$ and most did not [3].

However, the outcome of experimental haemarthrosis by injection of blood into the articular space was absorbance, rather than TMJ ankylosis [3, 5]. Similarly, the arthroscopic examination of 20 patients with acute TMJ trauma demonstrated that haemarthrosis rapidly spontaneously resolved by $5-7$ days [6]. These results indicated that haemarthrosis alone was not sufficient for the formation of ankylosis. Recently, we found that experimental haemarthrosis induced by discectomy resolved and no TMJ ankylosis occurred 3 months postoperatively in a sheep model $[7,8]$. However, if the discectomy combined with removal of the fibrous layers covering on the condyle and the glenoid fossa, haemarthrosis organized and developed into ankylosis inevitably [7].

Our findings suggested a couple of things. Firstly, since removal of the fibrous layers will lead to exposure of the underneath proliferative zone cells or chondrocytes on the articular surfaces according to the TMJ histology and our previous animal study [9], the fate of experimental haemarthrosis, whether absorbance or organization into ankylosis, depended on the tissue it contacted in the animal model. Secondly, although several previous studies had explored the molecular pathophysiology of TMJ ankylosis in animal models $[10,11]$, these studies, in fact, tried to answer how the haemarthrosis developed into ankylosis in a specific traumatic microenvironment, rather than elucidate the molecular basis why some of haemarthrosis organizated into ankylosis, while most of them resolved. Taking haematoma absorbance as a control to explore the molecular pathogenesis of TMJ ankylosis, would contribute to answering the question mentioned above. Thirdly, since most of haemarthrosis after TMJ trauma rapidly resolved within 1 week for human beings [6], taking haematoma absorbance as a control would also contribute to uncovering the early stage of molecular pathophysiology of TMJ ankylosis, which is largely unknown.

Therefore the experimental haemarthrosis with or without removal of articular fibrous layers is an established and suitable model for gene expression profiling studies regarding the onset of TMJ ankylosis. Our group has previously used a condylar fracture model to analyze the differential gene expression profiles between traumatic TMJ fibrous and bony ankylosis [12]. Recently, Zhang et al. [13] also explored the role of lateral pterygoid muscle in the traumatic TMJ ankylosis by gene chip. However, up to now there have been no published reports of genome-wide transcriptional analysis of TMJ ankylosis that takes place in an experimental haemarthrosis models without condylar fracture. Our aim in this study is to compare the differential gene transcription profiles between haematoma absorbance (HA) and haematoma organization ( $\mathrm{HO}$ ) into ankylosis in a sheep model of TMJ trauma. These findings will provide useful information for elucidating the molecular mechanism regarding the onset of TMJ ankylosis.

\section{Materials and methods}

\section{Animal model and tissue processing}

Three-month-old male small-tailed Han sheep with body weight ranging from 25 to $27 \mathrm{~kg}$ were used in this study under a research protocol approved by the Ethics Committee of Tianjin Stomatological Hospital (Tjskq2013001). The animals were kept in an animal-care facility with strict compliance to care and usage protocols that were previously described $[14,15]$. The animal model was established according to the protocol outlined in our previous publication [7]. The method of anesthesia, analgesia and euthanasia used for sheep was the same as described previously [12]. All animals received bilateral TMJ surgery. Disk removal was performed on one side, which has been shown to present as haematoma absorbance (HA) and the ensuing TMJ osteoarthritis, and removal of disk and articular fibrous layers was performed on the contralateral side, which has been shown to support hematoma organization $(\mathrm{HO})$ and produce TMJ fibro-osseous ankylosis [7].

Since the time for absorbance of haemarthrosis in the animal model was unclear, 15 sheep were sacrificed at days $1,4,7,9$ and 11 postoperatively with 3 animals being killed per time point to explore the early stage of pathological process in experiment 1 . After the sheep were sacrificed with a $120 \mathrm{mg} / \mathrm{kg}$ of pentobar-bitone sodium given through intravenous injection, the bilateral TMJs were opened carefully. The haematoma or newly formed tissue within the joint space was bluntly dissection from the surrounding soft tissue by periosteum separators. It is important to distinguish the haematoma located at the intracapsular or extracapsular site. Only the intracapsular haematoma or newly formed tissue was harvested for further analysis. The tissue was fixed in $10 \%$ natural buffered formalin for $72 \mathrm{~h}$, then dehydrated and embedded in paraffin. Five- $\mu \mathrm{m}$-thick slices were cut with a microtome, and the slides were examined after staining with hematoxylin-eosin (H-E).

According to the results of experiment 1 , another 9 sheep were added and sacrificed at days 1,4 and 7 after surgery with 3 animals being killed per time point in 
experiment 2. The site of tissue harvest was the same as experiment 1 . The tissue was rapidly frozen in liquid nitrogen and stored at $-80{ }^{\circ} \mathrm{C}$ for ribonucleic acid (RNA) extraction and the subsequent microarray analysis.

\section{RNA preparation and microarray data acquisition}

RNA preparation and microarray detection were performed by CNKingBio Corporation (Beijing, China). In brief, we extracted total RNA from the hematoma using Trizol reagent (Invitrogen Life Technologies, Carlsbad, USA) and using the RNEasy small kit (Qiagen, Valencia, USA) purified total RNA. Biotinylated complementary deoxyribonucleic acid (cDNA) was then prepared from 150 ng total RNA using an Ambion ${ }^{\circledR}$ WT expression kit according to the Affymetrix standard protocol. After tagging, fragments of cDNA were hybridized at $45{ }^{\circ} \mathrm{C}$ for $16 \mathrm{~h}$ on Affymetrix ${ }^{\circledR}$ Ovigene-1_0-ST Array (Affymetrix), a microarray containing over 22,047 known transcripts and probe sets of expressed sequence tags. The gene chip was cleaned and stained in the Affymetrix Fluidics Station 450. All arrays were scanned by the Affymetrix ${ }^{\circledR}$ Genchip Command Console (AGCC), which was installed in the Genchip ${ }^{\circledR}$ Scanner $30007 \mathrm{G}$.

\section{Microarray expression data analysis Identification of different expression genes (DEGs)}

The raw expression data were first background corrected and quantile normalized by the robust multichip analysis (RMA) algorithm using Affymetrix default analysis settings. Values presented were $\log _{2}$ RMA signal intensity. The fold-change (FC) was calculated with HA as control. DEGs were identified using limma package [16] in R/ Bioconductor (https://bioconductor.org/packages/relea se/bioc/html/limma.html) for comparison of the HA and $\mathrm{HO}$ with the cutoff value of $\mathrm{FC}>2$ and $p<0.05$. We used volcano plot and heatmap to show the distribution of DEGs intuitively. The volcano plot and heatmap of the DEGs were drawn via ggplot2 and heatmap packages in $R$ software [17].

\section{Function enrichment analysis}

The gene ontology (GO) term enrichment (http://www. geneontology.org/) was utilized to group the identified DEGs into defined categories of cellular component (CC), molecular function (MF) and biological process (BP) and determine which genes were significant [18]. Kyoto encyclopedia of genes and genomes (KEGG) PATHWAY (http://www.genome.jp/kegg/ pathway.html) was selected as the reference database for manually drawn pathway mapping [19]. The database for annotation, visualization and integration discovery (DAVID, version 6.8, http://david.abcc.Ncifcrf. gov/) [20] was recruited to perform the GO and KEGG pathway enrichment analysis and ascertained the functions and pathways that might be disturbed by the identified DEGs, with the selected criterion of a $p<0.05$.

\section{Protein-protein interaction network construction and module analysis}

To evaluate the interactive relationships among DEGs, the protein-protein interaction pairs were executed using the STRING (Search Tool for the Retrieval of Interacting Genes, version 11.0; https://string-db.org/) [18], which is an online database to assess and integrate physical and functional protein-protein associations with species limited to "Ovis aries" and an interaction score confidence $>0.4$ [21]. In PPI network, "node" represents a gene or protein, and "line" represents an interaction between the two nodes. The degree of each node (number of interactions with other proteins) is equal to the number of nodes that interacted with this node.

Significant modules in the PPI network were identified by molecular complex detection (MCODE) [22], a plug-in of Cytoscape software that clusters a network based on topology to recognize closely connected regions. The MCODE algorithm sorts and identifies each identified module. The higher the score is, the stronger the genes' association in this module. The parameters of DEGs clustering and scoring were set as follows: degree cutoff $=2$, node score cutoff $=0.2$, $\mathrm{K}$-Core $=2$, and max depth $=100$.

\section{Reverse transcription and real-time polymerase chain reaction ( $P C R$ )}

Six interesting DEGs were selected from the microarray results to further confirm the reliability of the array data. Total RNA was isolated using Trizol Reagent (Invitrogen Life Technologies, Carlsbad, USA) according to the manufacturer's instructions. Reverse transcription was performed with a cDNA synthesis kit (Promega, USA) in a $20 \mu \mathrm{l}$ reaction system containing $2 \mu \mathrm{g}$ total RNA [10].

Primers for each target gene were designed using the software of Primer Premier Version 5.0 and synthesized by Sangon Biotech (Shanghai) Co., Ltd, which were listed in Table 1. Quantitative real-time PCR was performed with FastStart Universal SYBR Green Master (Roche, ref. 04913850001) using LightCycler 480 II Instrument (Roche, Switzerland). The reaction system and PCR cycle parameters were the same as previously described [14]. The housekeeping glyceraldehyde-3-phosphate dehydrogenase (GAPDH) gene was used for normalization of target genes expression. Relative mRNA expressions of the target genes between the $\mathrm{HA}$ and $\mathrm{HO}$ were calculated using $2^{-\Delta \Delta c t}$ method as previously described [14]. 
Table 1 Real-time PCR primer sequence

\begin{tabular}{|c|c|c|c|}
\hline Gene & Gene bank number & Primer sequences $\left(5^{\prime}-3^{\prime}\right)$ & $\begin{array}{l}\text { Product } \\
\text { size (bp) }\end{array}$ \\
\hline \multirow[t]{2}{*}{ GAPDH } & AF030943.1 & GCAAGTTCCACGGCACAG & 249 \\
\hline & & GGTTCACGCCCATCACAA & \\
\hline \multirow[t]{2}{*}{$B G N$} & XM_015104664.2 & GGAAGGGTCTCTTGGGGTGC & 170 \\
\hline & & TCAAGGGGCATGTGATGGGG & \\
\hline \multirow[t]{2}{*}{ KDR } & XM_012179355.2 & ATGGGAACCGAAACCTA & 126 \\
\hline & & СCTGGGCACCTTCTACT & \\
\hline \multirow[t]{2}{*}{ POSTN } & XM_004012108.4 & $\begin{array}{l}\text { CCCCATAACTGTCTACAA } \\
\text { GCCA }\end{array}$ & 199 \\
\hline & & $\begin{array}{l}\text { TCTCACAGGTGTGTCTTC } \\
\text { TTGC }\end{array}$ & \\
\hline \multirow[t]{2}{*}{ CXCL12 } & XM_012105583.1 & CCTTGCCGATTCTTTGAG & 189 \\
\hline & & AGTGGGACTGGGTTTGTTT & \\
\hline \multirow[t]{2}{*}{ SPARC } & XM_012177565.3 & CTGGACTACATCGGGCCTTG & 156 \\
\hline & & CAGCTTCTGCTTCTCGGTCA & \\
\hline \multirow[t]{2}{*}{ TEK } & XM_012127828.2 & TCTGTGAAGGGCGAGTT & 196 \\
\hline & & GGCACCGAGTGGATGAA & \\
\hline
\end{tabular}

\section{Statistical analysis}

The values of the $\mathrm{HA}$ and $\mathrm{HO}$ were compared at each time point. Independent $t$ test was conducted using SPSS 17.0 (SPSS Inc., Chicago, IL) to determine whether statistical significance existed at a $p$ value $<0.05$.

\section{Results}

\section{Gross appearance of haematoma}

At days 1 and 4, large haematoma could be found by naked-eye in and around the joint cavities both in HA and $\mathrm{HO}$ sides (Fig. $1 \mathrm{~A}-\mathrm{H}$ ). At day 7 , the haematoma could also be observed in and around the joint cavities in the HO side (Fig. 1I, J). However the main part of haematoma in the HA side located at the extracapsule (Fig. $1 \mathrm{~K}, \mathrm{~L}$ ), indicating that it was almost absorbed in the joint space. At days 9 and 11, the haematoma in the $\mathrm{HO}$ side organized with the color changing from dark red to pink, and the new generated tissue filled in the lateral joint space (Fig. $1 \mathrm{M}, \mathrm{N}, \mathrm{Q}, \mathrm{R})$. While in the HA side, only a very small part of residual haematoma could be seen around the joint capsule but completely disappeared in the joint space without new generated tissue (Fig. 1O, P, S, T).

\section{Histological examination}

Due to the haematoma in the joint space was completely absorbed at days 9 and 11 in the HA side, we could only compare the histology between the $\mathrm{HA}$ and $\mathrm{HO}$ at days 1 , 4, and 7, and the results were illustrated in Fig. 2.
The first day after operation, blood clots filled the bilateral articular space, producing a fibrin and platelet laden with substrate for a succession of cellular infiltrates. Inflammatory cells were ubiquitous in the haematoma tissue, most of which were lobulated neutrophils. The degree of inflammation in the HA side was more severe than that in the HO side (Fig. 2A-D).

At day 4 after surgery, the haematoma was partially organized in the HO side. Besides erythrocytes and network of fibrin, a small amount of granulation tissue was found with blood vessels being detected (Fig. 2E, F). As a contrast, no granulation tissue was observed in the HA side, instead of a loose network of fibrin containing massive erythrocytes and mononuclear inflammatory cells (Fig. 2G, H).

At day 7 , the haematoma was highly organized by a fibrin scaffold and large amounts of granulation tissue, with angiogenesis and increased fibroblasts in the $\mathrm{HO}$ side (Fig. 2I, J). Surprisingly, granulation tissue was also found in the HA side although its range and size were much lower than that in the $\mathrm{HO}$ side (Fig. $2 \mathrm{~K}, \mathrm{~L}$ ). Combined with the gross appearance that the main part of haematoma in the HA side located at the extracapsule at day 7 , we speculated that the function of the granulation tissue was to repair the injured articular capsule.

\section{DEGs between $\mathrm{HO}$ and $\mathrm{HA}$ samples}

All the DEGs at days 1, 4 and 7 were showed in Additional files 1, 2 and 3. A total of 268 DEGs (117 up-regulated and 151 down-regulated) at day 1, 223 DEGs (91 up-regulated and 132 down-regulated) at day 4 and 17 DEGs (14 up-regulated and 3 down-regulated) at day 7 were found and illuminated by the volcano plots (Fig. 3A-C).

The heat maps using hierarchical clustering analysis showed that the expression patterns of mRNAs between the $\mathrm{HA}$ and $\mathrm{HO}$ samples were significantly different at day 1 (Fig. 3D), day 4 (Fig. 3E) and day 7 (Fig. 3F) postoperatively, which indicated that the tissue that the haematoma contacted with could determine the outcomes of the haematoma.

\section{GO and KEGG pathway analyses of DEGs}

The details of the GO and KEGG analysis results were presented in Additional files 4 and 5 .

At day 1, up-regulated DEGs could be enriched in 47 terms, including $28 \mathrm{BP}$ terms, $11 \mathrm{CC}$ terms and $8 \mathrm{MF}$ terms (Additional file 4). In the BP domain, the most meaningful enriched GO terms primarily focused on collagen fibril organization (GO:0030199), cartilage condensation (GO:0001502), and bone mineralization (GO:0030282) (Fig. 4A). The most enriched CC terms were correlated to extracellular structure, such 


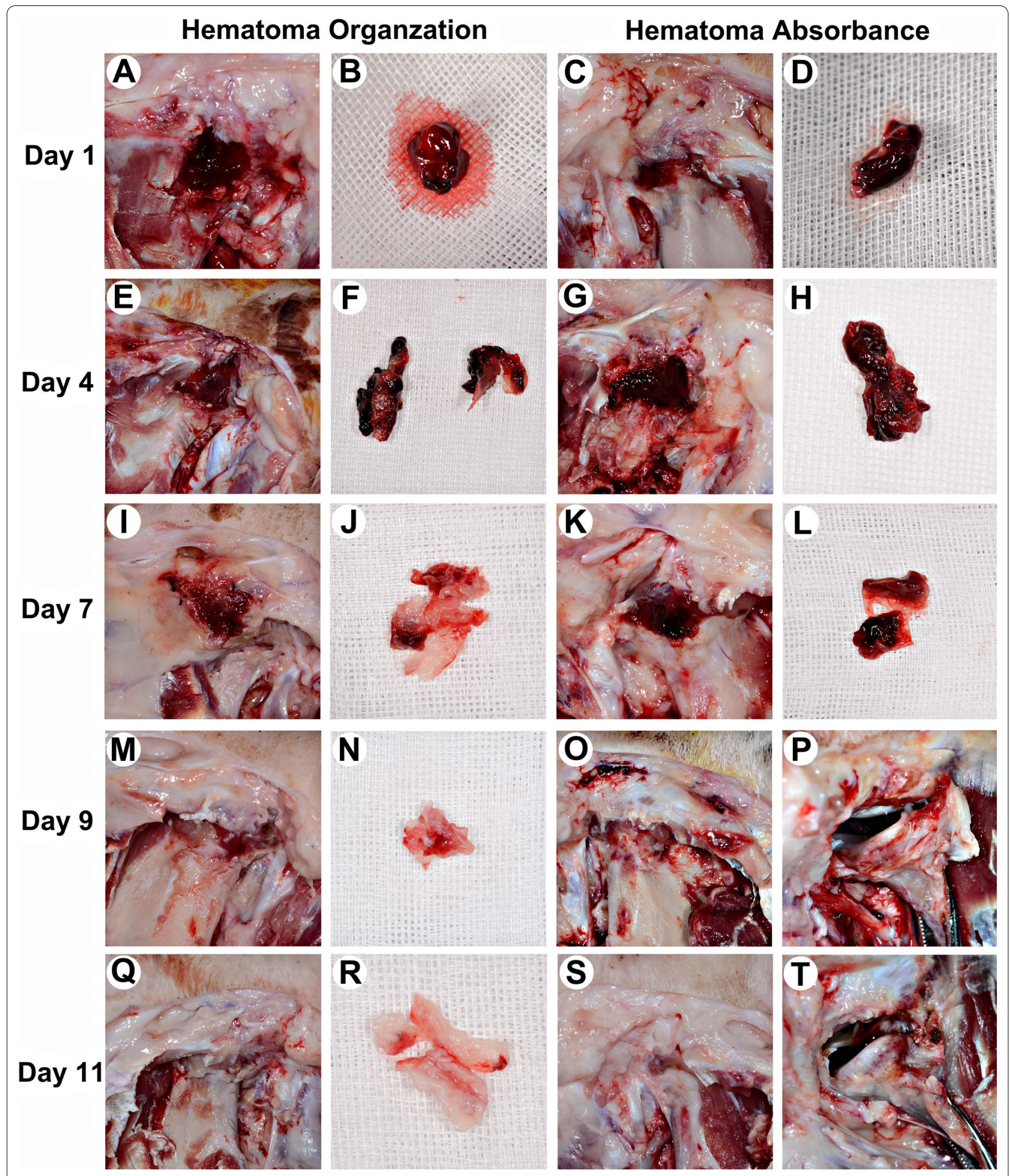

Fig. 1 The gross appearance of the temporomandibular joint and hematoma at each postoperative time. A-L At day 1, 4 and 7 postoperatively, the appearance of temporomandibular joint area and hematoma samples of $\mathrm{HO}$ and $\mathrm{HA}$ side. At these time points, bilateral intraarticular hematomas were all visible to the naked eye. $\mathbf{M}, \mathbf{N}, \mathbf{Q}, \mathbf{R}$ The performance of temporomandibular joint area and hematoma samples at day 9 and 11 of $\mathrm{HO}$ side. The hematoma has been organized on this side, and the new generated tissue filled in the joint space. $\mathbf{O}, \mathbf{P}, \mathbf{S}, \mathbf{T}$ The appearance of the temporomandibular joint area and how it looks when it's opened at day 9 and 11 of HA side, we can only see the empty temporal mandibular joint cavity without new generated tissue. HO hematoma origination, HA hematoma absorbance 


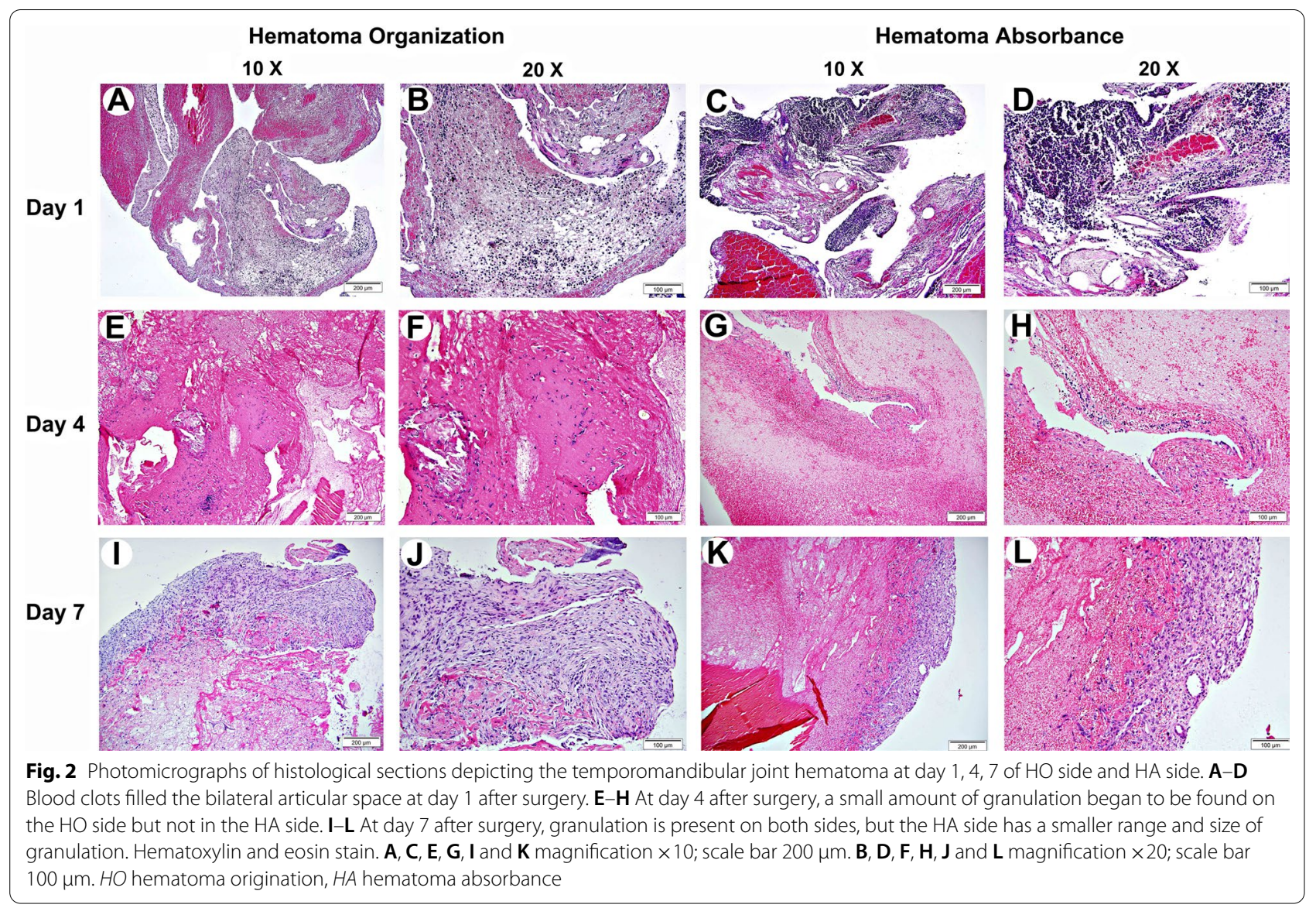

as extracellular space (GO:0005615), extracellular matrix (GO:0031012), and focal adhesion (GO:0005925) (Fig. 4A). For the MF terms, the represented terms were calcium ion binding (GO:0005509), heparin binding (GO:0008201), and extracellular matrix structural constituent activity (GO:0005201) (Fig. 4A). Furthermore, the KEGG pathway analysis indicated 10 KEGG pathways were significantly enriched and the up-regulated mRNAs were found to be associated with ECM-receptor interaction, Focal adhesion, PI3K-Akt signaling pathway and TGF-beta signaling pathway, Protein digestion and absorption (Fig. 4C).

Downregulated DEGs at day 1 could be enriched in 32 terms, including $23 \mathrm{BP}$ terms, $7 \mathrm{CC}$ terms and $2 \mathrm{MF}$ terms (Additional file 4). Figure 4B displayed the most significant BP, CC, MF terms. Among them, the most representative GO-BPs terms of these DEGs were mainly involved in the immune and inflammatory responses, such as immune response (GO:0006955), positive regulation of interleukin-12 production (GO:0032735), $\mathrm{T}$ cell receptor signaling pathway (GO:0050852), negative thymic $T$ cell selection (GO:0045060). At day 1, downregulated-DEGs significantly enriched in 17 pathways, and they were shown in bubble diagram (Fig. 4D), which were also almost related to immune response, such as Primary immunodeficiency, Cytokine-cytokine receptor interaction, Cell adhesion molecules, $\mathrm{T}$ cell receptor signaling pathway and Chemokine signaling pathway, etc.

At day 4, the 91 upregulated DEGs could be grouped into 33 terms including 17 in BP, 11 in CC and 5 in MF through $\mathrm{GO}$ analysis (Additional file 5), and listed in the histogram (Fig. 5A). The BP terms were most relevant to cell adhesion (GO:0007155), collagen fibril organization (GO:0030199), actomyosin structure organization (GO:0031032), negative regulation of cell proliferation (GO:0008285) and growth plate cartilage development (GO:0003417). The CC terms was similar to the 1-day enrichment result, which was also mostly enriched in extracellular structure. The MF terms were closely related to extracellular matrix structural constituent (GO:0005201), calcium ion binding (GO:0005509). KEGG analysis of the 91 DEGs identified 9 pathways, they were shown in bubble diagram (Fig. 5C). Those pathways that enriched at day 1 (ECM-receptor interaction, Focal adhesion, PI3K-Akt signaling pathway 

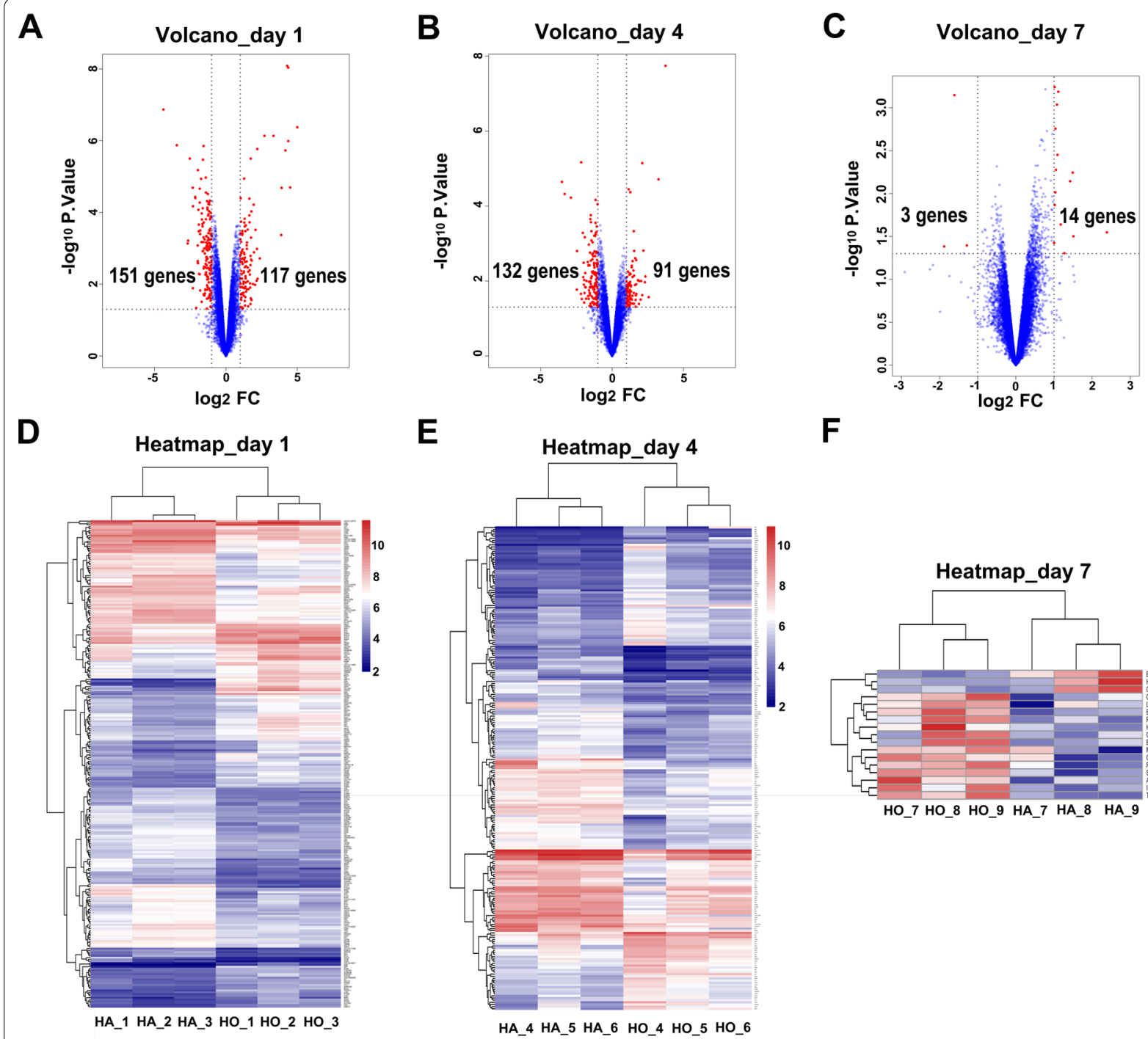

$\mathbf{F}$

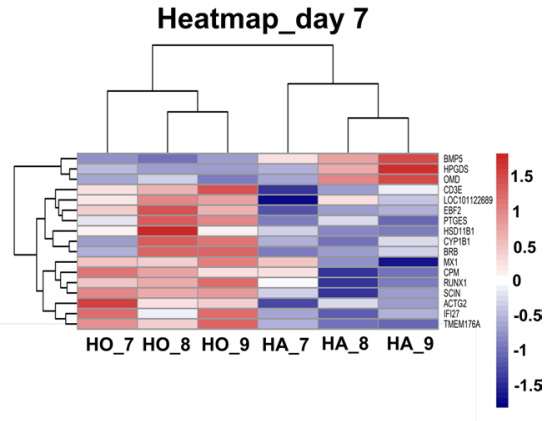

Fig. 3 DEGs (fold change $>2, p<0.05$ ) in the microarray profiles. A Volcano plot of 3 paired $\mathrm{HO}$ and HA samples at day 1. B Volcano plot of 3 paired $\mathrm{HO}$ and $\mathrm{HA}$ samples at day 4. C Volcano plot of 3 paired $\mathrm{HO}$ and HA samples at day 7. D Heat map of 3 paired $\mathrm{HO}$ and HA samples at day 1. E Heat map of 3 paired $\mathrm{HO}$ and $\mathrm{HA}$ samples at day 4. $\mathbf{F}$ Heat map of 3 paired $\mathrm{HO}$ and $\mathrm{HA}$ samples at day 7. Different colors represent different expression levels (red high expression, white medium expression, and blue low expression). DEGs different expression genes, HO hematoma origination, $H A$ hematoma absorbance

and TGF-beta signaling pathway, Protein digestion and absorption) were also enriched at day 4.

At day 4, the 132 down-regulated DEGs could be grouped into 66 terms including 47 in BP, 10 in CC and 9 in MF through GO analysis (Additional file 5), and the representative terms were listed in the histogram (Fig. 5B). The BP terms were most relevant to immune response (GO:0006955) and inflammatory response (GO:0006954). Meanwhile a lot of terms related to phagocytosis were enriched, such as receptor-mediated endocytosis (GO:0006898), MyD88-dependent toll-like receptor signaling pathway (GO:0002755), phagocytosis, engulfment (GO:0006911), cellular response to molecule of fungal origin (GO:0071226). KEGG analysis identified 16 pathways (Additional file 5 ), and the representative 10 pathways were shown in bubble diagram (Fig. 5D). Several pathways were interesting, for example, Phagosome, B cell receptor signaling pathway, Fc gamma R-mediated phagocytosis and NF-kappa B signaling pathway.

At day 7, statistically significant GO term and KEGG pathway were not enriched due to the low number of DEGs. 


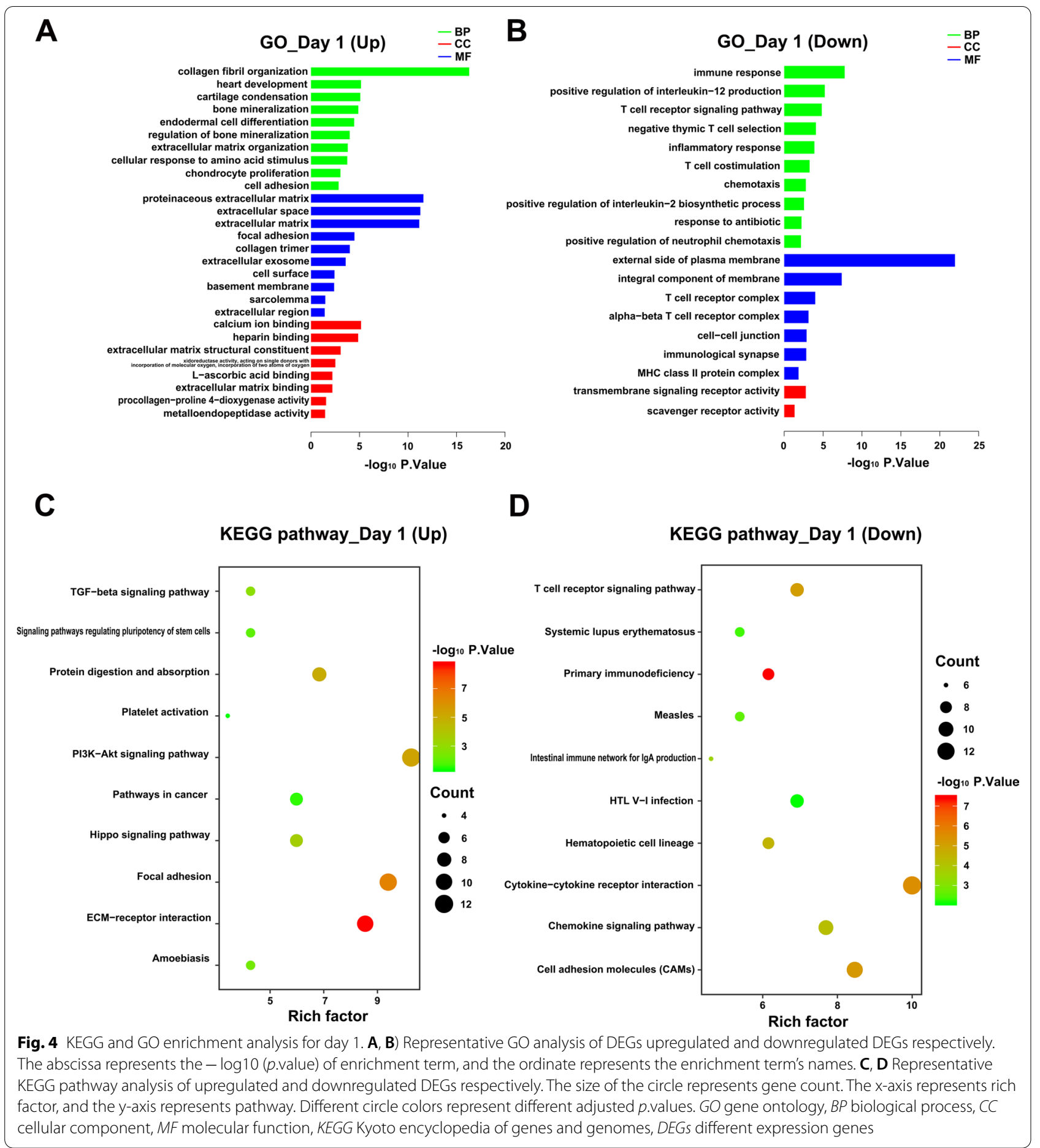

\section{PPI network and module analysis}

Based on the information of the STRING database, the PPI network of DEGs was constructed with 186 nodes and 614 edges (Fig. 6A) at day 1. By using MCODE analysis, 11 significant modules were screened out in the PPI network, and the most rewarding module
(MCODE score $=9.333)$ consisted of 10 nodes and 42 edges (Fig. 6B). The module comprised POSTN, BGN, SPARC, LUM, MMP2 and some genes encoded collagen (COL3A1, COL5A2, COL6A1, COL6A3 and COL11A1), which were all upregulated DEGs. The second rewarding module (MCODE score $=8.769$ ), consisting of 14 nodes 


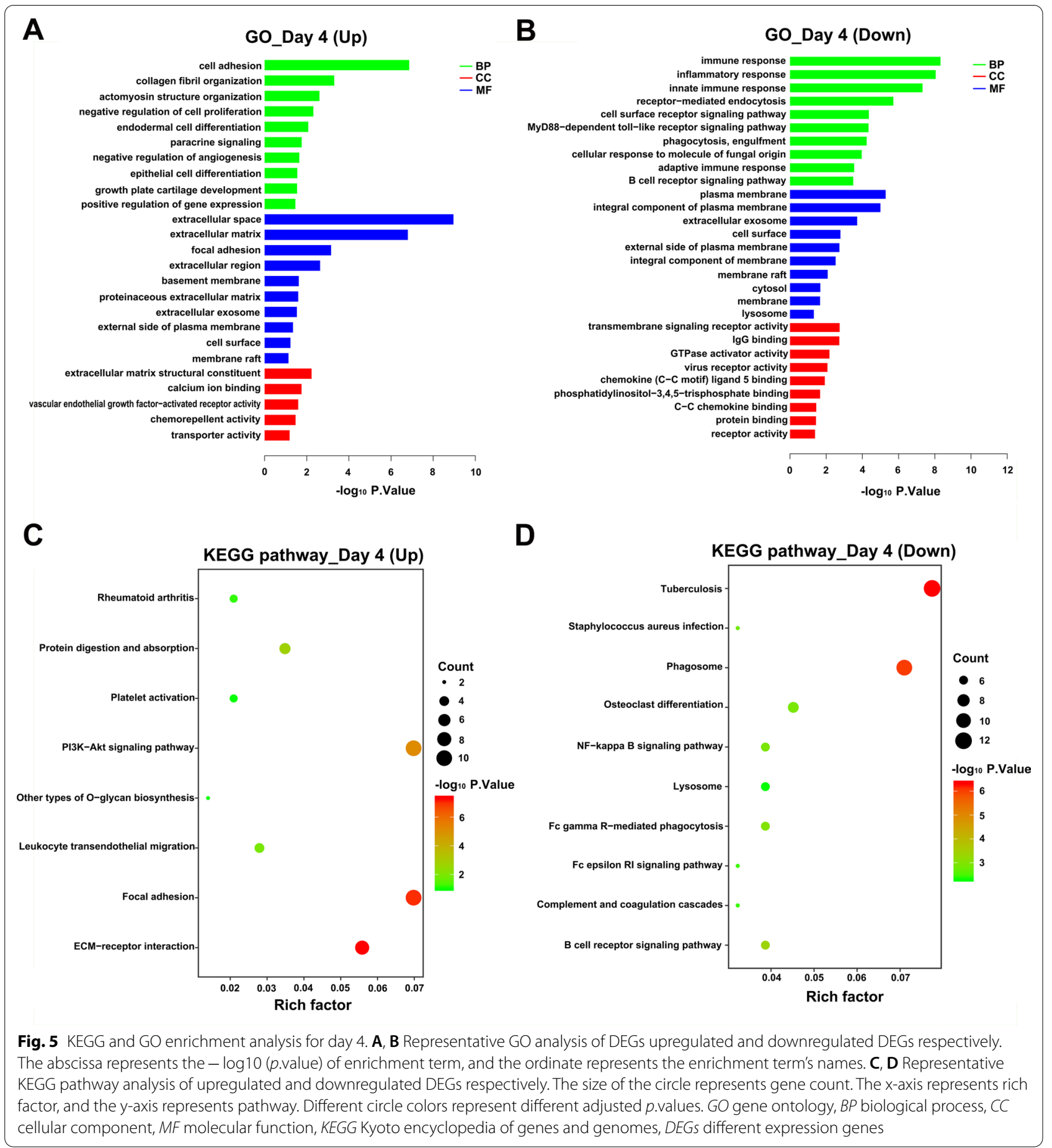

and 57 edges (Fig. 6C), was composed of genes involved in immunity, such as CD40, CD28, CXCR5, TNFRSF9, etc. Interestingly, they were all downregulated DEGs.

A total of 141 nodes and 356 edges were identified from the DEGs at day 4 (Fig. 7A). By using MCODE analysis, only 2 significant modules were screened out in the PPI network, and they were shown in Fig. 7B, C. The most rewarding module (MCODE score $=7.143$ ) consisted of 15 nodes and 50 edges (Fig. 7B). The genes in this module were all down-regulated DEGs, and mainly associated with interferon (IFIT1, IFI44L, IFI44, and MX2) and macrophage (MPEG1). The 


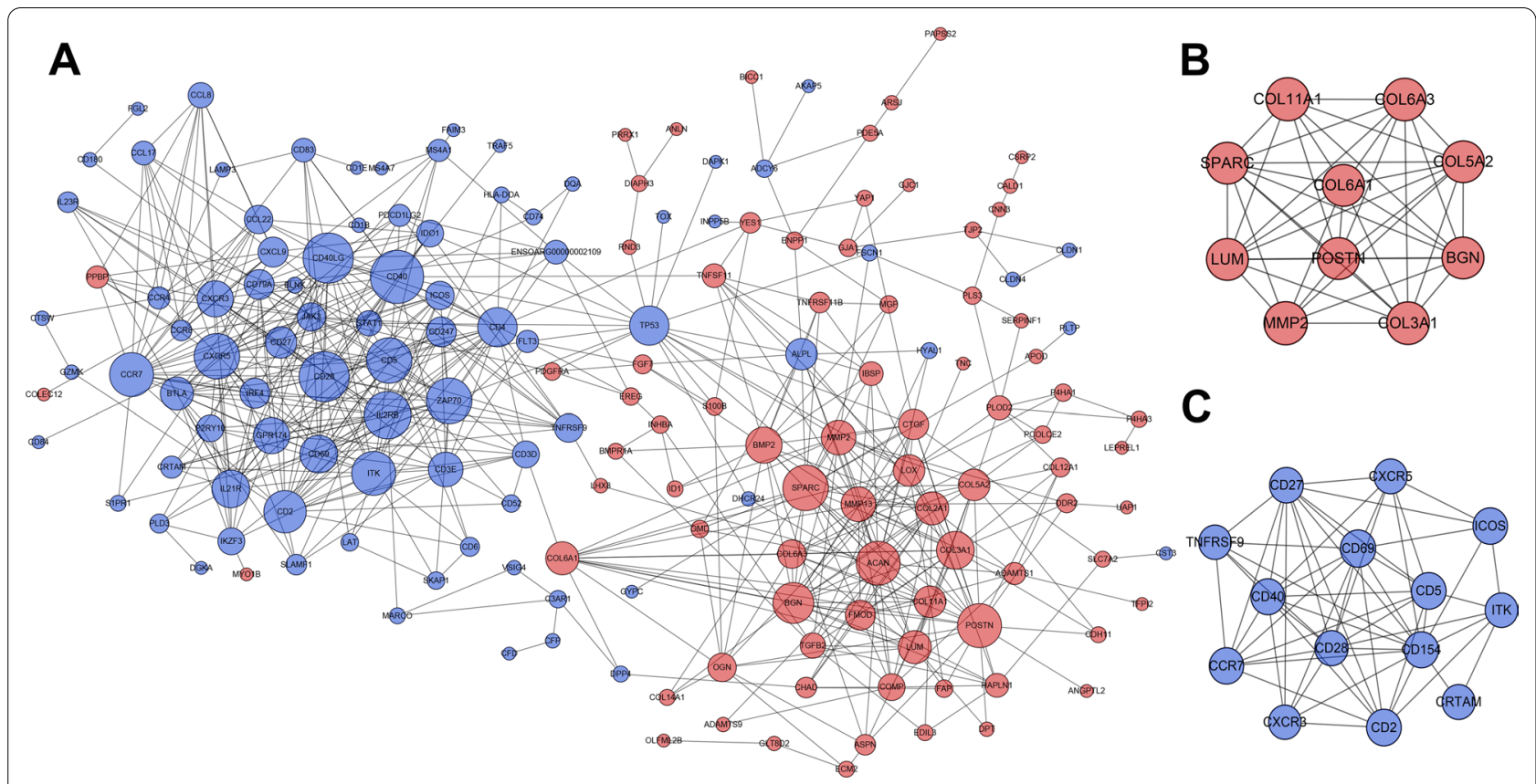

Fig. 6 PPI network construction and Module analysis of the DEGs at day 1. A The PPI network of the DEGs consisted of 186 nodes and 614 edges. B The most significant module consisted of 10 nodes and 42 edges extracted from PPI network (MCODE score $=9.333$ ). C The second significant module consisted of 14 nodes and 57 edges extracted from PPI network (MCODE score $=8.769$ ). The nodes represent proteins, and the edges between nodes represent interactions. The greater the degree of correlation, the larger the diameter of nodes. The nodes are red for upregulated DEGs, and blue for downregulated DEGs. PPI, protein-protein interaction; DEGs, different expression genes

second significant module was made up of both upregulated and downregulated DEGs (Fig. 7C). The upregulated DEGs in the module was mainly associated with angiogenesis, such as TEK, KDR, FIT1, and CXCL12. While the downregulated DEGs in the module were mainly related to the phagocytosis of macrophages, such as MRC1, CD163, CD68, and ITGAM.

\section{Verification of differentially expressed mRNAs by real time PCR}

Six interesting DEGs were selected for confirmation by quantitative real-time PCR analysis. All of these 6 DEGs were genes in significant modules according to PPI analysis. We found that the expression pattern of the 6 genes (Fig. 8) was similar to the microarray results, demonstrating a good correlation between the two methods.

At day 1, POSTN (10.1-fold, $p=0.026)$, BGN (3.9fold, $p=0.031$ ), and SPARC (2.7-fold, $p=0.021)$ were up-regulated in $\mathrm{HO}$ in comparison to HA (Fig. 8).

At day 4, HO showed a significantly higher expression of CXCL12 (1.9-fold, $p=0.001)$, TEK (2.0-fold, $p=0.026)$, and KDR (5.3-fold, $p=0.003)$, compared to HA (Fig. 8).

\section{Discussion}

Increasing evidences in TMJ ankylosis-related research have emphasized the importance of intra-articular haematoma, which possesses essential signals to initiate and activate healing processes $[5,23,24]$. In the present study, we explored the early pathological process of TMJ ankylosis in a sheep model by removal of disk and articular fibrous layers. The intra-articular haematoma organized into granulation tissue in the joint space at days 4 and 7 postoperatively. As a control, the blood clot in the joint space did not organize and was almost absorbed in the HA side at day 7 after surgery, which was in accordance with the arthroscopy of acute TMJ trauma of human beings [6].

The fate of experimental haemarthrosis, whether absorbance or organization into ankylosis, depended on the tissue it contacted in the sheep model. Namely, if haemarthrosis keeps in touch with the fibrous layers, it will be absorbed. While if haemarthrosis contacts and interactions with the proliferative zone cells or chondrocytes, ankylosis develops. The proliferation zone cells and articular chondrocytes might secrete cytokines and growth factors into the haematoma. Or the osteoprogenitor cells underneath the fibrous layers might migrate into the joint space mediated by the haematoma. These 

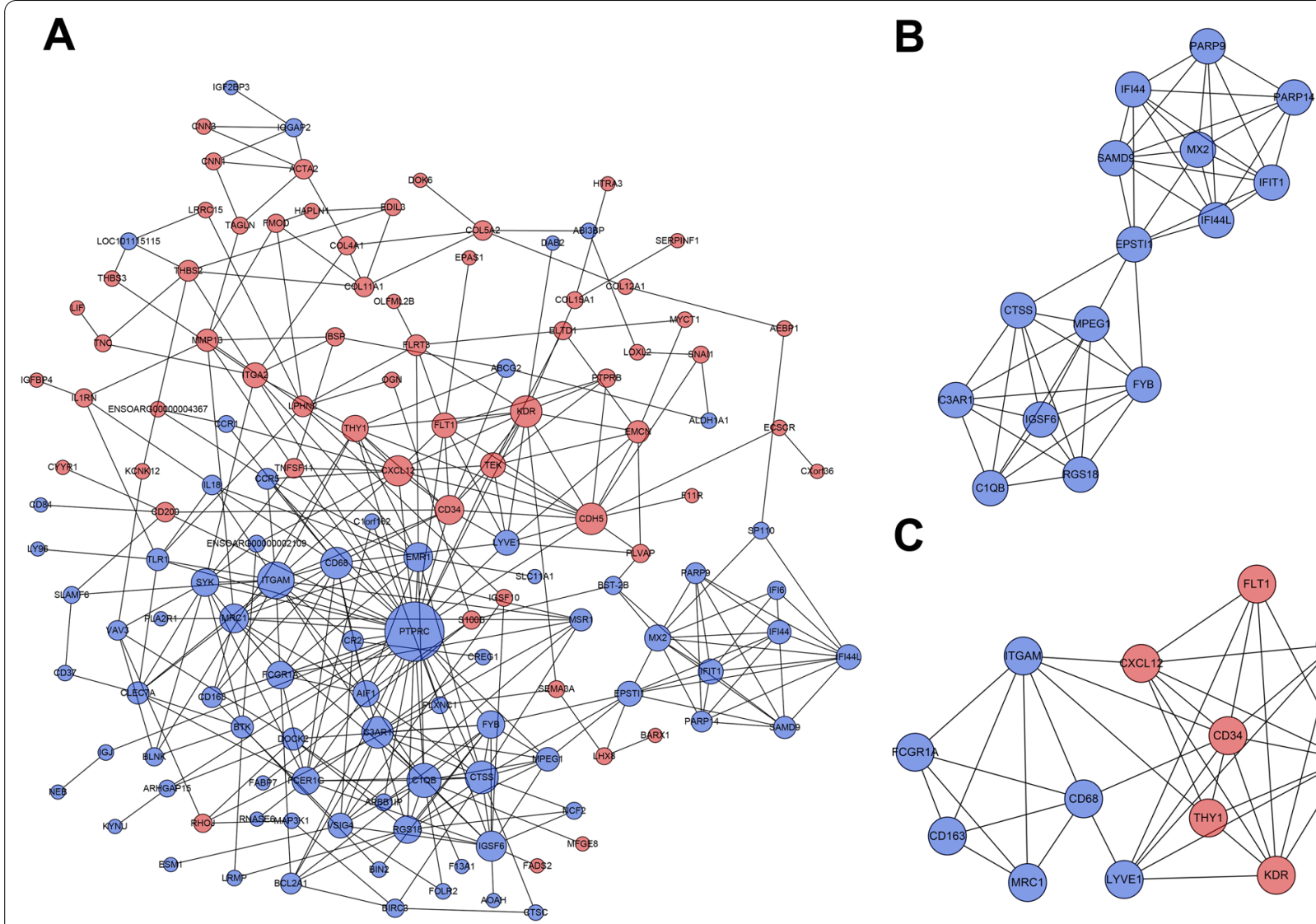

C

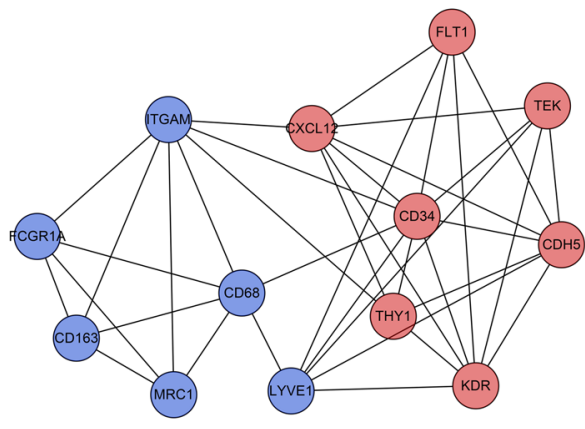

Fig. 7 PPI network construction and Module analysis of the DEGs at day 4. A The PPI network of the DEGs consisted of 141 nodes and 356 edges. B The most significant module consisted of 15 nodes and 50 edges extracted from PPI network (MCODE score $=7.143$ ). C The second significant module consisted of 13 nodes and 38 edges extracted from PPI network (MCODE score $=6.333$ ). The nodes represent proteins, and the edges between nodes represent interactions. The greater the degree of correlation, the larger the diameter of nodes. The nodes are red for upregulated DEGs, and blue for downregulated DEGs. PPI protein-protein interaction, DEGs different expression genes

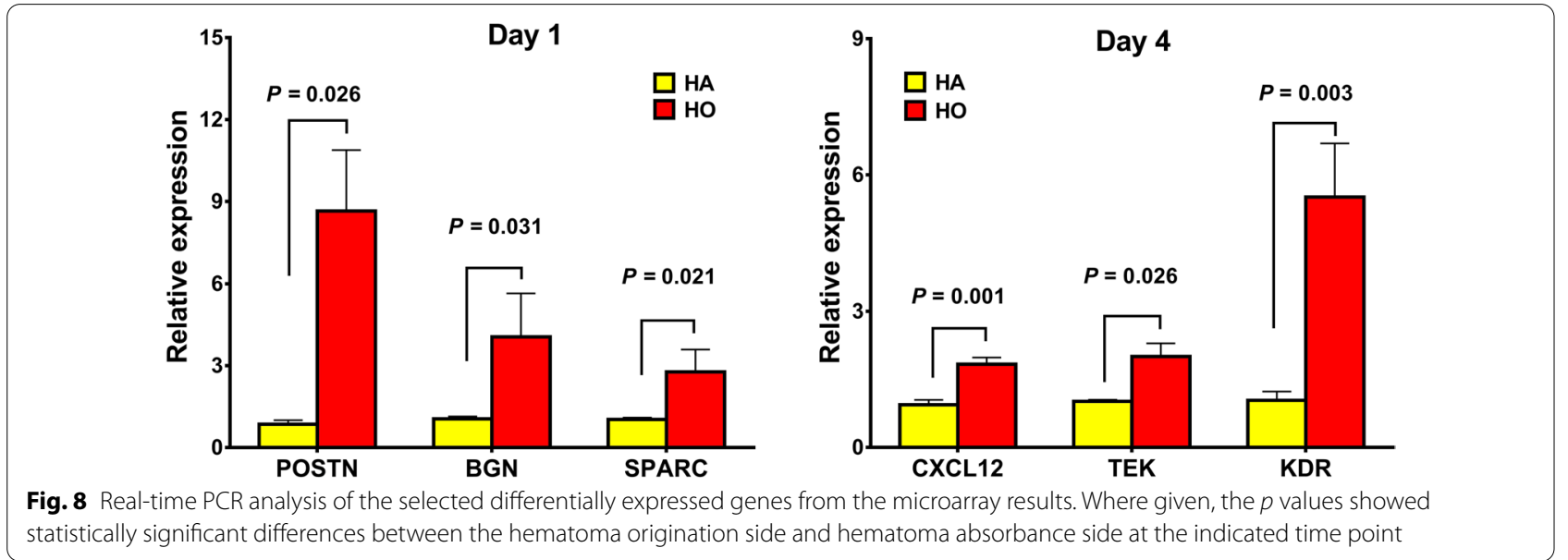

factors will promote the development of TMJ ankylosis. However, in the HA side, due to the barrier function of the fibrous layer, no foreign cells and growth factors can reach to and interact with the haematoma, therefore presenting with a natural course of haematoma absorbance. To explain the different outcomes of haematoma in the 
animal model, a genome-wide transcriptional analysis of haemarthrosis was performed, and a series of significant genes, pathways and molecular events during the onset and progress of TMJ ankylosis were identified.

A total of 268 and 223 DEGs between the $\mathrm{HO}$ and HA samples were screened at days 1 and 4 respectively. The large amount of DEGs at days 1 and 4 reflected the obviously differences between the haematoma absorbance and organization in the joint space. However only 17 DEGs were found at day 7. It seemed that there should also be a large number of DEGs at day 7 since the haemarthrosis was subsequently absorbed completely at days 9 and 11 in the disk removal side, while the hematoma was highly organized in the ankylosis-induced side at the same time point. The reason, we speculated, might be that the outcome of blood clot in the disk removal side was not simply absorbed in the joint space, but partly participated in the repair of the joint capsule. While the hematoma in the ankylosis-induced side not only repair of the joint capsule, but also organized in the joint space, therefore ultimately leading to TMJ ankylosis. Therefore the relatively less DEGs at day 7 indicated the similarity of repair of joint capsule between the 2 sides. The pathological results at day 7 confirmed our speculation.

At day 1 , as revealed by GO and KEGG pathway analysis, up-regulated DEGs in the ankylosis-induced side were enriched in the biological processes of collagen fibril organization and new bone formation, while at day 4 most overrepresented GO-BPs terms of up-regulated DEGs were mainly involved in cell adhesion, collagen fibril organization, and growth plate cartilage development. According to our previous study, the development of the TMJ ankylosis is similar to the mal-union of fracture [25]. In the present study, a large blood clot filled the joint space immediately after trauma due to the removal of the disc and the fibrous layers of the condylar head and glenoid fossa, as well as the tear of the attachment around the joint. Thrombase produced by blood vessel rupture induced fibrinogen in the blood to break down subsequently, forming fibrinogen monomers which are then rapidly aggregated. In this way, the earliest fibrin net, will be the first bridge among the traumatic articular surfaces within $6-8 \mathrm{~h}[26,27]$. As soon as a fibrin clot begins to form, mesenchymal stem cells (MSCs) will be recruited and give rise to fibroblasts. Once the fibroblasts are attached to the fibrin net, they secrete a large amount of collagen, including COL3A1, COL5A2, COL6A1, COL6A 3 and COL11A1 as shown in the present study, between 24 and $48 \mathrm{~h}$ after trauma [28]. In this collagen matrix, endothelial cells proliferate and gradually form new networks of capillaries. At the same time, TGF- $\beta$ pathway, which was produced by granulation tissue to regulate the proliferation of fibroblasts and collagen synthesis [29], was also highly expressed in the HO side. In addition, BMP- 2 , as one member of TGF- $\beta$ pathway, can also induce the further differentiation of stem cells into osteoblasts [30]. Meanwhile, apoptosis was inhibited following PI3-Akt pathway activation [31, 32]. Under the multiple effects of the above factors, the new tissue generated in the joint cavity.

Regarding the results in the HA side, we found a large number of neutrophils gathered at the surgical site within the first $24 \mathrm{~h}$ after surgery. BP terms such as immune response, $\mathrm{T}$ cell receptor signaling pathway, $\mathrm{T}$ cell costimulation and chemotaxis were enriched. Both histoligical and GO analysis showed that the HA side had a more severe inflammatory response than the HO side. Similarly, fibrin scaffolds and collagen matrix also formed in the joint space in the HA side. However, it seemed that they only served as scaffolding for the infiltrating cells (leukocytes, keratinocytes and fibroblasts) [33], since the intact articular surfaces prevented the key signaling factors regulating collagen mineralization and osteogenic differentiation of MSCs from entering the haematoma. More importantly, a large number of macrophages migrated to the site of injury and began to remove debris, necrotic tissue and pathogens at the site of injury during 48-96 $\mathrm{h}$ after surgery [34]. At this point, several interesting genes had higher expression in the HA side, for example, CD86 (promoting phagocytosis and removal of cell debris by macrophages [35]), MRC1 (promoting endosytosis of glycosylated protein and collagen in macrophages $[36,37]$ ), and CD163 (participating in phagocytosis and phagocytosis of hemoglobin/haptoglobin complex [38]). This also explained why the haematoma was absorbed from the molecular level.

In the present study, up-regulated DEGs in significant modules screened by PPI, such as POSTN, BGN, SPARC, and LUM at day 1 , and TEK, KDR, FLT1 at day 4, played an important role in this process above-mentioned.

At day 1, we found Periostin (POSTN) significantly up-regulated in the HA samples. Firstly identified in murine osteoblasts, this gene is now discovered to be specifically expressed in collagen-rich tissues, such as periodontal ligament and periosteum, and to transfer mechanical stress [39]. POSTN acts as a structural component of the matrix regulating collagen crosslinking and as a signaling molecule via interaction with integrin receptors and $\mathrm{Wnt} / \beta$-catenin pathways to promote osteoblast functions [40]. POSTN is considered a key extracellular matrix protein needed in healing. It also plays an important role in promoting periosteal callus formation and repair of bone fractures during the early stage of fracture healing [41]. In the present study, the up-regulated expression of POSTN in the HA at 
day 1 postoperatively indicated an important role in the onset of TMJ ankylosis, and deserved to be further studied.

Biglycan (BGN), Fibromodulin (FMOD) and lumican (LUM) are subtypes of the small leucine-rich family of proteoglycans (SLRP) [42]. SLRPs are the major noncollagen components of the extracellular matrix, with the function of affecting bone growth, craniofacial structure, dentin formation and collagen production [43]. Both $B G N$ and FMOD are critical for the differentiation and function of TMJ chondrocytes by modulating TGF- $\beta 1$ activity [44]. BGN deletion may cause structural abnormalities of bone collagen fibrils and impair the metabolic activity of bone marrow stromal cells (BMSCs) [45, 46]. Considering a role in early collagen fibril assembly [47], LUM might be involved in both inflammation and remodelling in response to dermal injury [48].

SPARC is a $32 \mathrm{kDa}$ calcium-binding matricellular protein, also referred to as osteonectin or basement membrane protein 40 (BM-40) [49]. During bone formation, SPARC is secreted by osteoblasts [50]. In osteoid, SPARC is thought to bind collagen and hydroxyapatite crystals and release calcium ions that promote the mineralization of the collagen matrix [51]. Notably, SPARC is expressed at high levels in bone remodeling process than steady bone tissue [52]. Delany et al. [53] have reported that osteoblastic precursors were reduced in SPARCnull mice, and that the capacity of osteoblast differentiation of bone marrow derived cells from SPARC-null was impaired.

All of the above molecules are expressed in the extracellular matrix and can participate in the reconstruction and assembly of collagen and affect the next step of bone mineralization, suggesting that extracellular matrix plays an important role in the occurrence of TMJ ankylosis. The presumption is in accordance with the results of CC in our $\mathrm{GO}$ analysis.

At day 4, the genes regulating angiogenesis (TEK, KDR, FLT1) were screened by PPI analysis. The process of angiogenesis is mainly regulated by two molecular pathways. One is angiopoietin-dependent pathway, the other is vascular endothelial growth factor (VEGF)-dependent pathway [54]. Their expression was induced early in the healing cascade, suggesting that they promoted initial vascular growth of existing vessels in the periosteum [54]. TEK (also known as Tie2) is the tyrosine kinase receptor of Angiopoietin-1 (Ang 1) and Angiopoietin-2 (Ang 2) in the angiotensin signaling pathway [55]. VEGF binds to tyrosine kinase cell receptors, including VEGFR-1 (Flt1), and VEGFR-2 (KDR). VEGFR-1 and VEGFR-2 are expressed predominantly on vascular endothelial cells, with strong pro-angiogenic activity [56]. These results suggest that angiogenesis was significantly enhanced at day 4 in the $\mathrm{HO}$ side, which is also consistent with our histological results.

Among the up-regulated genes screened by PPI analysis in the ankylosis-induced side at day 4, a key gene, CXCL12, is worth noting. It is a key inflammatory cytokine and may be involved in the initial inflammatory response as the first step of fracture healing. As an important chemokine, CXCL12 has been identified to regulate the inflammatory response associated with the healing process [57]. Furthermore, it is an important contributor to bone marrow MSC homing and localization within the bone marrow [58]. Our findings indicated that the early expression of CXCL12 could promote the genesis of TMJ ankylosis, therefore might be an promising target for the prevent of TMJ ankylosis in the future.

This study has some limitations. First, the number of experimental animals is too small, which may lead to an increase in type 2 errors in statistics. Secondly, the whole-body circulation of cytokines/inflammation response might affect bilateral injury sites since the ipsand contra-lateral tissue samples were obtained from the same animal. However, the animal model was reliable, and the obvious histological differences between the HA and $\mathrm{HO}$ side was confirmed in the present study. Therefore, the molecular basis underlying the histological differences, as uncovered by microarray analysis of DEGs, was convincing. Finally, the key genes and signaling pathways obtained based on bioinformatics methods are only a preliminary study, and further basic experiments and clinical validation are needed in the future.

In conclusion, our study demonstrated the DEGs between the $\mathrm{HO}$ and HA samples at several time points postoperatively by using an extensive bioinformatics analysis. Genes that promoted collagen ossification (POSTN, BGN, LUM, SPARC) and angiogenesis (KDR, FIT1, TEK) had a positive effect on the onset and progress of TMJ ankylosis. Further investigation of the precise functional roles of these genes will provide new ideas for future treatment and prevention of the disease.

\footnotetext{
Abbreviations

TMJ: Temporomandibular joint; HA: Haematoma absorbance; HO: Haematoma organization; DEGs: Different expression genes; PPI: Protein-protein interaction; FC: Fold-change; GO: Gene ontology; BP: Biological process; MF: Molecular function; CC: Cellular component; DAVID: Database for annotation visualization and integrated discovery; KEGG: Kyoto encyclopedia of genes and genomes; STRING: Search tool for the retrieval of interacting genes/proteins; MCODE: Molecular complex detection; PCR: Reverse transcription and real-time polymerase chain reaction; GAPDH: Glyceraldehyde-3-phosphate dehydrogenase; POSTN: Periostin; BGN: Biglycan; SPARC: Secreted protein acidic and cysteine rich; LUM: Lumican; MMP2: Matrix metallopeptidase 2; COL3A1: Collagen type III alpha 1 chain; COL5A2: Collagen type V alpha 2 chain; COL6A1: Collagen type VI alpha 1 chain; COL6A3: Collagen type VI alpha 3 chain; COL11A1: Collagen type XI alpha 1 chain; CD40: CD40 molecule; CD28: CD28 antigen; CXCR5: C-X-C motif chemokine receptor 5; TNFRSF9: TNF receptor superfamily member 9; IFIT1: Interferon induced protein with
} 
tetratricopeptide repeats 1; IFI44L: Interferon induced protein 44 like; IFI44: Interferon induced protein 44; MX2: MX dynamin like GTPase 2; MPEG1: Macrophage expressed 1;TEK: TEK receptor tyrosine kinase; KDR: Kinase insert domain receptor; FIT1: FMS related receptor tyrosine kinase 1; CXCL12: C-X-C motif chemokine ligand 12; MRC1: Mannose receptor C-type 1; CD163: CD163 molecule; CD68: CD68 molecule; ITGAM: Integrin subunit alpha M; MSCs: Mesenchymal stem cells; BMP-2: Bone morphogenetic protein 2; TGF- $\beta$ : Transforming growth factor, beta; FOMD: Fibromodulin; SLRP: Small leucinerich family of proteoglycans; VEGF: Vascular endothelial growth factor; Ang 1: Angiopoietin-1; Ang 2: Angiopoietin-2.

\section{Supplementary Information}

The online version contains supplementary material available at https://doi. org/10.1186/s12903-021-02033-w.

$$
\begin{aligned}
& \text { Additional file 1. DEGS at day } 1 . \\
& \text { Additional file 2. DEGS at day } 4 . \\
& \text { Additional file 3. DEGS at day } 7 \text {. } \\
& \text { Additional file 4. GO and KEGG pathway analyses of DEGS at day } 1 .
\end{aligned}
$$

Additional file 5. GO and KEGG pathway analyses of DEGS at day 4.

\section{Acknowledgements}

No.

\section{Authors' contributions}

$\mathrm{M}-\mathrm{NJ}$ and $\mathrm{T}-\mathrm{MZ}$, participated in the design of the experiment, were responsible for conceptualization, methodology, investigation, writing the main text, figures, tables and revising the draft critically for important intellectual content and agreement to be accountable for all aspects of the work. Kun Yang was responsible for methodology, investigation, writing the main text and agreement to be accountable for all aspects of the work. Z-YX and G-MZ were responsible for methodology, investigation and agreement to be accountable for all aspects of the work. Y-YT was responsible for investigation and agreement to be accountable for all aspects of the work did the experiment and data analysis. Y-BY and $\mathrm{HL}$ were responsible for conceptualization, methodology, writing-reviewing and editing the main manuscript, figures, tables, also the supervision of work. All authors read and approved the final manuscript.

\section{Funding}

This investigation was supported by Tianjin Key Discipline Foundation of Medicine.

\section{Availability of data and materials}

The datasets used and/or analyzed during the current study are available from the corresponding author on reasonable request.

\section{Declarations}

\section{Ethics approval and consent to participate}

Animal studies were approved by the medical ethics committee of Tianjin Stomatological Hospital and all procedures were performed by the Regulations of the Animal Management Regulations and Administrative Measures on Experimental Animal. All studies involving animals were reported in accordance with the ARRIVE guidelines for reporting experiments involving animals.

\section{Consent for publication}

Not applicable.

\section{Competing interests}

The authors declare that they have no competing interests.

\section{Author details}

${ }^{1}$ Tianjin Medical University, 22 Qi-xiang-tai Road, Heping District, Tianjin 300070, People's Republic of China. ${ }^{2}$ Department of Oromaxillofacial-Head and Neck Surgery, Tianjin Stomatological Hospital, 75 Dagu Road, Heping District, Tianjin 300041, People's Republic of China. ${ }^{3}$ Tianjin Key Laboratory of Oral and Maxillofacial Function Reconstruction, 75 Dagu Road, Heping District, Tianjin 300041, People's Republic of China. ${ }^{4}$ Department of Oral and Maxillofacial Surgery, China Three Gorges University Affiliated Renhe Hospital, 410 Yiling Ave, Hubei 443001, People's Republic of China.

Received: 26 August 2021 Accepted: 22 December 2021

Published online: 28 December 2021

\section{References}

1. Valentini V, Vetrano S, Agrillo A, Torroni A, Fabiani F, lannetti G. Surgical treatment of TMJ ankylosis: our experience (60 cases). J Craniofac Surg. 2002;13(1):59-67.

2. Yan Y, Zhang Y, Sun Z, Li J, Xiao E, An J. The relationship between mouth opening and computerized tomographic features of posttraumatic bony ankylosis of the temporomandibular joint. Oral Surg Oral Med Oral Pathol Oral Radiol Endod. 2011;111(3):354-61.

3. Laskin DM. Role of the meniscus in the etiology of posttraumatic temporomandibular joint ankylosis. Int J Oral Surg. 1978;7(4):340-5.

4. Hall MB. Condylar fractures: surgical management. J Oral Maxillofac Surg. 1994;52(11):1189-92.

5. Oztan HY, Ulusal BG, Aytemiz C. The role of trauma on temporomandibular joint ankylosis and mandibular growth retardation: an experimental study. J Craniofac Surg. 2004;15(2):274-82 (discussion 82).

6. Goss AN, Bosanquet AG. The arthroscopic appearance of acute temporomandibular joint trauma. J Oral Maxillofac Surg. 1990;48(8):780-3 (discussion 4).

7. Wang HL, Liu H, Shen J, Zhang PP, Liang SX, Yan YB. Removal of the articular fibrous layers with discectomy leads to temporomandibular joint ankylosis. Oral Surg Oral Med Oral Pathol Oral Radiol. 2019;127(5):372-80.

8. Yang K, Wang HL, Dai YM, Liang SX, Zhang TM, Liu H, Yan YB. Which of the fibrous layer is more important in the genesis of traumatic temporomanibular joint ankylosis: the mandibular condyle or the glenoid fossa? J Stomatol Oral Maxillofac Surg. 2020;121(5):517-22.

9. Wang HL, Zhang PP, Meng L, Liang SX, Liu H, Yan YB. Preserving the fibrous layer of the mandibular condyle reduces the risk of ankylosis in a sheep model of intracapsular condylar fracture. J Oral Maxillofac Surg. 2018:76(9):19511 e1-e24.

10. Yan YB, Li JM, Xiao E, An JG, Gan YH, Zhang Y. A pilot trial on the molecular pathophysiology of traumatic temporomandibular joint bony ankylosis in a sheep model. Part I: expression of Wnt signaling. J Craniomaxillofac Surg. 2014;42(2):e15-22

11. Yan YB, Li JM, Xiao E, An JG, Gan YH, Zhang Y. A pilot trial on the molecular pathophysiology of traumatic temporomandibular joint bony ankylosis in a sheep model. Part II: The differential gene expression among fibrous ankylosis, bony ankylosis and condylar fracture. J Craniomaxillofac Surg. 2014;42(2):e23-8.

12. Zhang DM, Yang $K$, Liang $S X$, Zhang PP, Wang HL, Tian YY, Xu ZY, Liu H, Yan YB. Microarray analysis of differential gene expression between traumatic temporomandibular joint fibrous and bony ankylosis in a sheep model. Med Sci Monit. 2021;27:e932545.

13. Zhang J, Sun X, Jia S, Jiang X, Deng T, Liu P, Hu K. The role of lateral pterygoid muscle in the traumatic temporomandibular joint ankylosis: a gene chip based analysis. Mol Med Rep. 2019:19(5):4297-305.

14. Liang SX, Wang HL, Zhang PP, Shen J, Yang K, Meng L, Liu H, Yan YB. Differential regulation of blood vessel formation between traumatic temporomandibular joint fibrous ankylosis and bony ankylosis in a sheep model. J Craniomaxillofac Surg. 2019:47(11):1739-51.

15. Yan YB, Zhang Y, Gan YH, An JG, Li JM, Xiao E. Surgical induction of TMJ bony ankylosis in growing sheep and the role of injury severity of the glenoid fossa on the development of bony ankylosis. J Craniomaxillofac Surg. 2013;41(6):476-86.

16. Ritchie ME, Phipson B, Wu D, Hu Y, Law CW, Shi W, Smyth GK. limma powers differential expression analyses for RNA-sequencing and microarray studies. Nucleic Acids Res. 2015;43(7):e47.

17. Yi G, Liang M, Li M, Fang X, Liu J, Lai Y, Chen J, Yao W, Feng X, Hu, et al. A large lung gene expression study identifying IL1B as a novel player in airway inflammation in COPD airway epithelial cells. Inflamm Res. 2018;67(6):539-51. 
18. Harris MA, Clark J, Ireland A, Lomax J, Ashburner M, Foulger R, Eilbeck K, Lewis S, Marshall B, Mungall C, et al. The gene ontology (GO) database and informatics resource. Nucleic Acids Res. 2004;32(Database issue):D258-61.

19. Kanehisa M, Goto S. KEGG: Kyoto encyclopedia of genes and genomes. Nucleic Acids Res. 2000;28(1):27-30.

20. Huang DW, Sherman BT, Tan Q, Collins JR, Alvord WG, Roayaei J, Stephens R, Baseler MW, Lane HC, Lempicki RA. The DAVID gene functional classification tool: a novel biological module-centric algorithm to functionally analyze large gene lists. Genome Biol. 2007;8(9):R183.

21. Szklarczyk D, Franceschini A, Wyder S, Forslund K, Heller D, Huerta-Cepas J, Simonovic M, Roth A, Santos A, Tsafou KP, et al. STRING v10: proteinprotein interaction networks, integrated over the tree of life. Nucleic Acids Res. 2015;43(Database issue):D447-52.

22. Bader GD, Hogue CW. An automated method for finding molecular complexes in large protein interaction networks. BMC Bioinform. 2003:4:2.

23. Norholt SE, Krishnan V, Sindet-Pedersen S, Jensen I. Pediatric condylar fractures: a long-term follow-up study of 55 patients. J Oral Maxillofac Surg. 1993;51(12):1302-10.

24. Yan YB, Liang SX, Shen J, Zhang JC, Zhang Y. Current concepts in the pathogenesis of traumatic temporomandibular joint ankylosis. Head Face Med. 2014;10:35.

25. Yan YB, Duan DH, Zhang Y, Gan YH. The development of traumatic temporomandibular joint bony ankylosis: a course similar to the hypertrophic nonunion? Med Hypotheses. 2012;78(2):273-6.

26. Schmidt-Bleek K, Schell H, Schulz N, Hoff P, Perka C, Buttgereit F, Volk HD, Lienau J, Duda GN. Inflammatory phase of bone healing initiates the regenerative healing cascade. Cell Tissue Res. 2012;347(3):567-73.

27. Gerstenfeld LC, Cullinane DM, Barnes GL, Graves DT, Einhorn TA. Fracture healing as a post-natal developmental process: molecular, spatial, and temporal aspects of its regulation. J Cell Biochem. 2003:88(5):873-84.

28. Echeverri LF, Herrero MA, Lopez JM, Oleaga G. Early stages of bone fracture healing: formation of a fibrin-collagen scaffold in the fracture hematoma. Bull Math Biol. 2015;77(1):156-83.

29. Clark RA, Nielsen LD, Welch MP, MCPherson JM. Collagen matrices attenuate the collagen-synthetic response of cultured fibroblasts to TGF-beta. J Cell Sci. 1995;108(Pt 3):1251-61.

30. Chen XJ, Shen YS, He MC, Yang F, Yang P, Pang FX, He W, Cao YM, Wei QS. Polydatin promotes the osteogenic differentiation of human bone mesenchymal stem cells by activating the BMP2-Wnt/beta-catenin signaling pathway. Biomed Pharmacother. 2019;112:108746.

31. Luo HR, Hattori H, Hossain MA, Hester L, Huang Y, Lee-Kwon W, Donowitz M, Nagata E, Snyder SH. Akt as a mediator of cell death. Proc Natl Acad Sci U S A. 2003;100(20):11712-7.

32. Ding ML, Ma H, Man YG, Lv HY. Protective effects of a green tea polyphenol, epigallocatechin-3-gallate, against sevoflurane-induced neuronal apoptosis involve regulation of CREB/BDNF/TrkB and PI3K/Akt/ mTOR signalling pathways in neonatal mice. Can J Physiol Pharmacol. 2017:95(12):1396-405.

33. Gantwerker EA, Hom DB. Skin: histology and physiology of wound healing. Facial Plast Surg Clin N Am. 2011;19(3):441-53.

34. Rodero MP, Khosrotehrani K. Skin wound healing modulation by macrophages. Int J Clin Exp Pathol. 2010;3(7):643-53.

35. Shinjo R, Imagama S, Ito Z, Ando K, Nishida Y, Ishiguro N, Kadomatsu K. Keratan sulfate expression is associated with activation of a subpopulation of microglia/macrophages in Wallerian degeneration. Neurosci Lett. 2014;579:80-5.

36. Kato M, Neil TK, Fearnley DB, McLellan AD, Vuckovic S, Hart DN. Expression of multilectin receptors and comparative FITC-dextran uptake by human dendritic cells. Int Immunol. 2000;12(11):1511-9.

37. Taylor PR, Gordon S, Martinez-Pomares L. The mannose receptor: linking homeostasis and immunity through sugar recognition. Trends Immunol. 2005;26(2):104-10.

38. di Masi A, De Simone G, Ciaccio C, D'Orso S, Coletta M, Ascenzi P. Haptoglobin: from hemoglobin scavenging to human health. Mol Aspects Med. 2020:73:100851

39. Horiuchi K, Amizuka N, Takeshita S, Takamatsu H, Katsuura M, Ozawa H, Toyama Y, Bonewald LF, Kudo A. Identification and characterization of a novel protein, periostin, with restricted expression to periosteum and periodontal ligament and increased expression by transforming growth factor beta. J Bone Miner Res. 1999;14(7):1239-49.
40. Bonnet N, Garnero P, Ferrari S. Periostin action in bone. Mol Cell Endocrinol. 2016;432:75-82.

41. Nakazawa T, Nakajima A, Seki N, Okawa A, Kato M, Moriya H, Amizuka N, Einhorn TA, Yamazaki M. Gene expression of periostin in the early stage of fracture healing detected by cDNA microarray analysis. J Orthop Res. 2004;22(3):520-5.

42. Schaefer $L$, lozzo RV. Biological functions of the small leucine-rich proteoglycans: from genetics to signal transduction. J Biol Chem. 2008:283(31):21305-9.

43. Bi Y, Stuelten CH, Kilts T, Wadhwa S, lozzo RV, Robey PG, Chen XD, Young MF. Extracellular matrix proteoglycans control the fate of bone marrow stromal cells. J Biol Chem. 2005;280(34):30481-9.

44. Embree MC, Kilts TM, Ono M, Inkson CA, Syed-Picard F, Karsdal MA, Oldberg A, Bi Y, Young MF. Biglycan and fibromodulin have essential roles in regulating chondrogenesis and extracellular matrix turnover in temporomandibular joint osteoarthritis. Am J Pathol. 2010;176(2):812-26.

45. Corsi A, XuT, Chen XD, Boyde A, Liang J, Mankani M, Sommer B, lozzo RV, Eichstetter I, Robey PG, et al. Phenotypic effects of biglycan deficiency are linked to collagen fibril abnormalities, are synergized by decorin deficiency, and mimic Ehlers-Danlos-like changes in bone and other connective tissues. J Bone Miner Res. 2002;17(7):1180-9.

46. Chen XD, Shi S, XUT, Robey PG, Young MF. Age-related osteoporosis in biglycan-deficient mice is related to defects in bone marrow stromal cells. J Bone Miner Res. 2002;17(2):331-40.

47. Ezura Y, Chakravarti S, Oldberg A, Chervoneva I, Birk DE. Differential expression of lumican and fibromodulin regulate collagen fibrillogenesis in developing mouse tendons. J Cell Biol. 2000;151(4):779-88.

48. Miragliotta V, Raphael K, Lussier JG, Theoret CL. Equine lumican (LUM) CDNA sequence and spatio-temporal expression in an experimental model of normal and pathological wound healing. Vet Dermatol. 2009:20(4):243-8

49. Murphy-Ullrich JE, Sage EH. Revisiting the matricellular concept. Matrix Biol. 2014:37:1-14.

50. Holland PW, Harper SJ, McVey JH, Hogan BL. In vivo expression of mRNA for the Ca++-binding protein SPARC (osteonectin) revealed by in situ hybridization. J Cell Biol. 1987;105(1):473-82.

51. Termine JD, Kleinman HK, Whitson SW, Conn KM, McGarvey ML, Martin GR. Osteonectin, a bone-specific protein linking mineral to collagen. Cell. 1981;26(1 Pt 1):99-105.

52. Delany AM, Hankenson KD. Thrombospondin-2 and SPARC/osteonectin are critical regulators of bone remodeling. J Cell Commun Signal. 2009:3(3-4):227-38

53. Delany AM, Kalajzic I, Bradshaw AD, Sage EH, Canalis E. Osteonectin-null mutation compromises osteoblast formation, maturation, and survival. Endocrinology. 2003;144(6):2588-96.

54. Marsell R, Einhorn TA. The biology of fracture healing. Injury. 2011:42(6):551-5

55. Ai-Aql ZS, Alagl AS, Graves DT, Gerstenfeld LC, Einhorn TA. Molecular mechanisms controlling bone formation during fracture healing and distraction osteogenesis. J Dent Res. 2008;87(2):107-18.

56. Melincovici CS, Bosca AB, Susman S, Marginean M, Mihu C, Istrate M, Moldovan IM, Roman AL, Mihu CM. Vascular endothelial growth factor (VEGF) — key factor in normal and pathological angiogenesis. Rom J Morphol Embryol. 2018;59(2):455-67.

57. Galliera E, Corsi MM, Banfi G. Platelet rich plasma therapy: inflammatory molecules involved in tissue healing. J Biol Regul Homeost Agents. 2012;26(2 Suppl 1):35S-42S.

58. Yellowley C. CXCL12/CXCR4 signaling and other recruitment and homing pathways in fracture repair. Bonekey Rep. 2013;2:300.

\section{Publisher's Note}

Springer Nature remains neutral with regard to jurisdictional claims in published maps and institutional affiliations. 